\title{
Personalidade e Coping em Pacientes com Transtornos Alimentares e Obesidade
}

\author{
Personality and Coping in Patients with Eating Disorders and Obesity
}

\author{
Renata Tomaz* \& Daniela S. Zanini \\ Pontifícia Universidade Católica de Goiás
}

\begin{abstract}
Resumo
Este estudo avalia o uso diferencial de coping e traço de personalidade em pacientes com transtornos alimentares (anorexia, bulimia e TASOE) e com obesidade e em população geral. Participam deste estudo 109 indivíduos (60 com diagnóstico de transtorno alimentar ou obesidade e 49 da população geral). Os instrumentos foram uma escala de traços de personalidade, Coping Response Inventory e Escala de Atitudes Alimentares (EAT). Observou-se diferença significativa nas médias de EAT por população demonstrando boa adequação deste instrumento como screening psicopatológico de transtornos alimentares. Ademais indivíduos que apresentam alto índice em neuroticismo e em descarga emocional, ao enfrentar seus problemas, possuem mais atitudes alimentares inadequadas refletidas pelo EAT $(R=0.291, p=0.011)$. Os dados são discutidos através das teorias relacionadas aos cinco grandes traços da personalidade, coping, transtornos alimentares e obesidade.
\end{abstract}

Palavras-chave: Traço de personalidade; Coping; Transtornos alimentares; Obesidade.

\begin{abstract}
This study assesses the differential use of coping and personality trait of patients with eating disorders (anorexia, bulimia, and Eating Disorders Not Otherwise Specified - EDNOS), obesity as well as in subjects from the general population. 109 subjects participated in the study (60 with eating disorder or obesity diagnostics; 49 from the general population). The instruments were Personality Trait Scale, Coping Response Inventory and Eating Attitudes Scale (EAS). It was observed significant differences on EAS according to the type of population, demonstrating this instrument's adequacy as psychopathological screening for eating disorders. Moreover, individuals presenting high neuroticism and who discharge their emotion to cope with their problems have more inadequate eating attitudes as shown by $\mathrm{EAS}(R=0.291$, $p=0.011$ ). These results are discussed through theories related to the Big Five personality traits, coping, eating disorders and obesity.

Keywords: Personality trait; Coping; Eating disorders; Obesity.
\end{abstract}

As doenças relacionadas com a alimentação atualmente têm despertado maior interesse e preocupação. Isso se deve à proliferação de patologias, como transtornos alimentares (anorexia, bulimia, Transtorno Alimentar Sem Outra Especificação) e obesidade (Cordás \& Weinberg, 2002; Hay, 2002; Magalhães \& Mendonça, 2005). A sintomatologia dessas enfermidades, ainda que aparentemente divergentes, possuem semelhanças, visto que, em geral, estão acompanhadas de comportamentos como ingestão compulsiva de alimentos, ingestão de grande quantidade de alimento em pequeno espaço de tempo, falta de controle alimentar e sentimento de culpa (Pinheiro, Sullivan, Bacaltchuck, Padro-Lima, \& Bulik, 2006).

Além disso, na anorexia, na bulimia e no Transtorno Alimentar Sem Outra Especificação, os indivíduos apresentam similarmente uma percepção distorcida da ima-

* Endereço para correspondência: Mestrado em Psicologia Pontifícia Universidade Católica de Goiás. Av. Universitária, 1069, St. Universitário, Caixa Postal 86, CEP74605010. Tef.: 3946-1116. E-mail: rrtomaz@gmail.com gem corporal, utilizam formas extremas para perder peso (usam medicamentos impróprios, induzem vômitos, fazem jejuns prolongados e praticam exercícios físicos em excesso) e apresentam elevada insatisfação com o próprio corpo, tudo isso acompanhado do medo intenso de engordar (Binford, Crosby, Mitchell, Mussel, Peterson, Crow, 2005).

Além dos transtornos alimentares, outra doença também associado à alimentação, contudo identificado como uma entidade nosológica distinta, é a obesidade. Esta é abordada neste artigo como excesso de peso que ultrapassa o índice de massa corporal (IMC) de $30 \mathrm{~kg} / \mathrm{m}^{2}$ (Teichmann, Olinto, Costa, \& Ziegler, 2006). Em termos epidemiológicos, pode-se dizer que o número de obesos aumentou significativamente. Isso fez da obesidade uma epidemia mundial, o que resultou na sua identificação como uma doença de etiologia multicausal. No Brasil, estudos realizados por Dobrow, Kamenetz e Devlin (2002) têm demonstrado que houve um aumento de $70 \%$ no número de pessoas classificadas como obesas, entre 1975 e 1989. 
As consequiências geradas pela obesidade também merecem atenção especial, Velásquez-Meléndez, Gazzinelli, Côrrea-Oliveira, Pimenta e Kac (2007) comprovaram que o excesso de peso está diretamente relacionado a doenças crônicas, como as cardiovasculares, patologias que atingem, atualmente, o maior índice de mortalidade. Outros danos causados por essa doença são os psicossociais, em virtude do estigma e da discriminação que indivíduos obesos sofrem (Teichmann et al., 2006). Além disso, King, Shapiro, Helb, Singletary e Turner (2006) e Shapiro, King e Quiñones (2007) apontam que esse preconceito contribui para o surgimento de psicopatologias, como depressão e transtornos ansiosos.

A importância do estudo dos transtornos alimentares e da obesidade está não somente no fato de sua alta incidência na sociedade moderna, mas também nos índices de mortalidade e morbidade relacionados a seu diagnóstico (Pinheiro, et al., 2006; Pitanga \& Lessa, 2007). De acordo com dados da American Psychiatric Association ([APA], 2002), em um estudo de acompanhamento de pacientes, após 10 anos de diagnóstico dos transtornos alimentares, a mortalidade acompanha $10 \%$ dos casos e, após 20 anos, esse índice sobe para $20 \%$.

Os números alarmantes das estatísticas referentes aos transtornos alimentares e à obesidade provocaram uma maior preocupação a respeito dessas patologias, com grande interesse em sua etiologia. Porém, sabe-se que o surgimento dessas doenças não é unideterminado, ao contrário, é multicausal, tendo como base fatores socioculturais, biológicos e psicológicos (Dobrow et al., 2002; Gorgati, Holcberg, \& Oliveira, 2002).

A seriedade e a complexidade dessas doenças demandam pesquisas e técnicas de intervenção ativas nessa área (Bighetti, Santos, \& Ribeiro, 2006; Wilson \& Fairburn, 1993). Estudos recentes demonstram a eficiência das estratégias de coping, entendido como esforços cognitivos e comportamentais realizados pelos indivíduos para fazer frente a situações apreciadas como estressantes (Lazarus \& Folkman, 1984), como forma terapêutica em relação a diversas patologias, incluindo os transtornos alimentares e a obesidade.

Além disso, a relação entre saúde física, mental e coping é bastante abordada atualmente (Gómez-Fraguela, Luengo-Martín, Romero-Triñanes, Villar-Torres, \& Sobral-Fernández, 2006). Estudos comprovam que estratégias de coping desadaptadas podem contribuir para o desenvolvimento de doenças em geral, assim como para a manutenção destas (Jonge \& Dormann, 2006; Schat, Kelloway, \& Desmarais, 2005). Mais especificamente, há evidências que o coping está associado a transtornos alimentares e obesidade (Galaif, Sussman, Chou, \& Wills, 2003; Gregor, 2005; Griffith, Dubow, \& Ippolito, 2000).

O estilo de estratégias que determinada pessoa utiliza pode influenciar na manifestação de doenças como transtornos psiquiátricos, como citam Margis, Picon, Cosner e Silveira (2003). Assim, ao vivenciar situações estressantes, os indivíduos podem desenvolver transtor- nos psiquiátricos, como estresse pós-traumático e sintomas depressivos e ansiosos, dependendo do estilo de coping utilizado. Por tanto, pode-se avaliar a relação entre coping e saúde pela forma como as estratégias estão relacionadas ao bem-estar físico e mental (Blumenthal, Babyak, Carney, Keefe, Davis, \& LaCaille, 2006; Sorkin \& Rook, 2006).

Demandas pessoais, como fatores da personalidade, podem influenciar a forma como as pessoas reagem a situações difíceis (Furtado, Falcone, \& Clark, 2003; Rowe, 2006). Por exemplo, o traço de personalidade neuroticismo parece preceder estratégias de enfrentamento, como descarga emocional, que, por sua vez, interfere no bem-estar do indivíduo (Connor-Smith \& Flachsbart, 2007).

Além disso, também são descritos usos diferenciais de estratégias de coping segundo características dos transtornos. Por exemplo, estudos em população clínica demonstram que esta tende a utilizar mais coping evitativo (Guedea, Albuquerque, Tróccoli, Noriega, Seabra, \& Guedea, 2006), assim como pacientes com transtornos alimentares (Rebelo \& Leal, 2007) e com obesidade (Rydén, Karlsson, Persson, \& Sjöström, 2001).

Fyer, Waller e Stenfert-kroese (1997) realizaram diversas pesquisas em pacientes com transtornos alimentares. Correlacionando a psicopatologia com coping, estresse e personalidade, verificaram que, esses indivíduos apresentam maior pontuação em neuroticismo e baixa adesão ao tratamento (Binford et al., 2005; Gongora, van de Staak, \& Derksen, 2004), o que, por sua vez, pode ser ainda mais comprometedor e auxiliar no incremento dos índices de morbidade e mortalidade destes. Nesse sentido, este artigo pretende avaliar o uso diferencial de coping e o perfil psicológico de pacientes com transtornos alimentares (anorexia, bulimia e TASOE) e com obesidade e da população geral.

\section{Método}

\section{Participantes}

Participaram deste estudo 109 individuos. Destes 60 eram pacientes de uma clínica escola sendo 30 sujeitos com transtornos alimentares e 30 sujeitos com obesidade, os outros 49 sujeitos eram provenientes da população geral.

Como critérios de inclusão foram solicitados que a população clínica apresentasse: diagnóstico de transtornos alimentares e obesidade, derivados de médicos psiquiatras e endocrinologistas; os critérios diagnósticos APA (1995) e da Organização Mundial de Saúde ([OMS], 1993); e idade acima de 17 anos. Os critérios de exclusão consistiam em pacientes com mais de mais de seis meses de tratamento psicoterápico e psiquiátrico, e ter histórico de drogadição.

A população geral foi constituída de 49 sujeitos, préselecionados em três instituições de ensino superior do mesmo município, que apresentassem critérios como: 
IMC entre 20 e $25 \mathrm{Kg} / \mathrm{m}^{2}$, obtidos através da medição do peso e da altura de todos estes sujeitos, no caso de IMC entre 25 a $30 \mathrm{Kg} / \mathrm{m}^{2}$ nesta amostra foi considerado como fator de risco para obesidade; e ter idade acima de 17 anos. Determinaram-se como critérios de exclusão ter histórico de drogadição e de transtornos mentais e alimentares.

\section{Instrumentos}

Foram utilizados para este estudo uma escala para avaliação da personalidade baseada na teoria do Big Five versão brasileira experimental, o Coping Response Inventory - Adult Form (CRI - A) e o Eating Attitudes Test (EAT-26).

A escala da personalidade foi utilizada para avaliar dois fatores: neuroticismo e extroversão da amostra deste estudo. Paralelamente, realizou-se a caracterização da medida para que se pudesse utilizá-la. Este teste foi baseado no Inventário Fatorial da Personalidade criado por Pasquali, Azevedo e Ghesti (1997), que avalia 15 características psicológicas.

Os fatores subdividem-se em 25 frases que possuem escalas Likert, as quais variam de 1 (extremamente incaracterística) a 5 (extremamente característica). Os itens foram precedidos por um parágrafo que solicitava aos participantes que expressassem o seu grau de concordância com cada uma das afirmações contidas na escala. Para esse estudo, foi realizada uma análise exploratória, a fim de se investigar o valor discriminativo da escala de personalidade, seguindo os procedimentos para a construção e a elaboração de instrumentos de avaliação de construtos psicológicos (Dessen, 2005; Tróccoli, Vasconcelos, Araújo, \& Meiçó, 2001). Avaliaram-se as agrupações derivadas da análise fatorial dos itens identificados, teórica e metodologicamente, como dois fatores: extroversão e neuroticismo. A validação da escala de personalidade foi realizada por intermédio do programa SPSS 13.0, através da análise fatorial. Inicialmente utilizou-se a análise de PC (Principal Components) com rotação varimax, eigenvalues superior a 1,4 e carga fatorial mínima de 0,40 para inclusão dos itens. Verificou-se a fatorabilidade dos dados disponíveis $(\mathrm{KMO}=$ 0,70 ; comunalidades variando de 0,26 a 0,67 ); os resultados desta análise inicial demonstraram a estrutura fatorial composta por cinco fatores.

Reproduziu-se a análise fatorial empregando-se o método PAF (Principal Axis Factoring) com rotação oblimin (método oblíquo). Foi estipulado o critério de carga fatorial mínima 0,40 e eigenvalues superior a 1,4. Os itens que não adquiriram a carga fatorial estipulada foram excluídos. Dessa forma, os fatores que apresentam melhores valores estatísticos foram neuroticismo e extroversão, por isso serão abordados neste estudo. Para avaliar as estratégias de coping, empregou-se o Coping Response Inventory Adult Form (CRI - A), de Moos (1993). Esse inventário está composto por 48 perguntas acerca de como o individuo enfrenta um problema des- crito anteriormente. As perguntas são respondidas por meio de uma escala Likert de 4 pontos que se agrupam posteriormente, por meio da adição direta dos itens em oito escalas de estratégias de coping. Oito tipos de estratégias de coping diretas se agrupam posteriormente em dois conjuntos descritos como coping aproximativo e coping evitativo segundo o foco do problema. As estratégias de enfrentamento de aproximação possuem a finalidade de solucionar a situação estressante, de modo semelhante ao coping focalizado no problema (analise lógica, reavaliação positiva, busca de guia, resolução de problemas). Por outro lado, as estratégias evitativas buscam controlar as emoções e/ou os pensamentos sobre o problema, sem enfrentar a situação de risco, como o coping centrado na emoção (evitação cognitiva, aceitação/resignação, busca de recompensas alternativas e descarga emocional). O CRI-A é um instrumento que possui grande renome internacional e apresenta uma base teórica consistente com sua aplicação metodológica (Moos, 2002, 2003). Sua versão brasileira está em fase de adaptação, porém dados preliminares apontam caracteristicas psicométricas similares a do instrumento original com pontuações alfa das escalas oscilando entre 0,68 e 0,72 .

Para avaliar comportamentos e atitudes alimentares próprias de pessoas que sofrem de transtornos alimentares, foi utilizado o Eating Attitudes Test (EAT-26). Este instrumento apresenta boa qualidade psicométrica e tem sido utilizado em diversos estudos para avaliar comportamentos relativos a transtornos alimentares assim como critério diagnóstico para os mesmos (Cordás \& Neves, 2000). Neste estudo utilizou-se a versão reduzida, que contém 26 itens, divididos em três escalas: da dieta, de comportamentos bulímicos e preocupação com os alimentos e de controle oral (Freitas, Gorenstein, \& Appolinario, 2002).

Os itens são pontuados por meio de uma escala de 0 a 3, em que as respostas "sempre", "muitas vezes" e "às vezes pontuam" 3,2 e 1, respectivamente, mas as respostas "poucas vezes", "quase nunca" e "nunca" não conferem pontuação. Dessa forma, os indivíduos que atingem pontuação acima de 21 são classificados como indivíduos com comportamentos referentes a distúrbio alimentar (Nunes, Appolinario, Abuchaim, \& Coutinho, 2006).

\section{Procedimento}

Mediante contato com médicos psiquiatras e endocrinologistas, solicitou-se o encaminhamento de pessoas que atendessem aos critérios diagnósticos para transtornos alimentares (anorexia, bulimia ou TASOE) e para obesidade ao serviço de psicologia de uma clínica escola. Uma vez feito o primeiro contato com os pacientes encaminhados e obtida a sua aprovação para a pesquisa, realizavam-se a leitura e a assinatura do Termo de Consentimento Livre e Esclarecido. Realizaram-se dois encontros individuais, de aproximadamente 50 minutos 
cada, com cada participante, nas próprias instalações da clínica escola. No primeiro encontro, foi aplicada a escala de personalidade e, no segundo, aplicaram-se os demais instrumentos.

Para a coleta de dados com a amostra da população geral foi realizado o contato com instituições de ensino superior e, uma vez obtida a autorização para realizar a pesquisa, fez-se o contato com os participantes, a assinatura do Termo de Consentimento Livre e Esclarecido e procedeu-se à aplicação dos questionários. Estes foram aplicados em dois momentos de 50 minutos cada, na própria sala de aula do participante, em situação coletiva. A todos os participantes do estudo foi garantido a anonimidade, assim como a possibilidade de retirada do consentimento a qualquer momento do processo, sem que sofressem dano algum. Também lhes foi oferecida a possibilidade de maiores explicações acerca do estudo em andamento, a qualquer momento, por meio de contato com a pesquisadora responsável.

\section{Resultados}

Neste trabalho, a análise dos dados foi realizada por meio do pacote estatístico para Windows SPSS versão 13.0. A amostra deste estudo foi constituída de $27,5 \%$ sujeitos com transtornos alimentares e $27,5 \%$ sujeitos com obesidade, a população geral foi constituída de $45 \%$. A idade destes variou entre 17 e 59 anos, com média de $30,57(D P=11,314)$. A maioria dos sujeitos da amostra total era solteira (56\%), naturais de Goiânia $(36,7 \%)$, cursava o ensino superior $(61,5 \%)$ e possuía índice de massa corpórea média de $26,95 \mathrm{~kg} / \mathrm{m}^{2}(D P=9,288)$.

Os resultados serão apresentados em duas sessões. $\mathrm{Na}$ primeira, os dados relativos à descrição da amostra e comparação de média dos traços de personalidade (neuroticismo e extroversão), estratégias de coping e atitudes alimentares segundo o tipo de população (geral ou com transtornos alimentares ou obesidade). Na segunda, o estudo correlacional de todas as variáveis estudadas e posteriormente o estudo de regressão linear dos traços de personalidade e estratégias de enfrentamento sobre as atitudes alimentares.

\section{Estudo Descritivo e Comparação de Média}

entre Personalidade, Coping e Atitudes Alimentares

Segundo o Tipo de População

Com o objetivo de avaliar a existencia de diferenças significativas nas pontuações médias dos traços de personalidade neuroticismo e extroversão, estratégias de coping e escalas do EAT segundo o tipo de população estudada (geral X com transtorno alimentar com obesidade) foi realizado um estudo de comparação de médias através da ANOVA. A Tabela 1 apresenta os resultados.

Tabela 1

Descritivos e Comparação de Média entre Fatores da Personalidade, Estratégias de Coping e Escalas do EAT-26 por Tipo de População

\begin{tabular}{|c|c|c|c|c|c|}
\hline \multirow[t]{3}{*}{ Variáveis sociodemográficas } & \multicolumn{5}{|c|}{ População } \\
\hline & \multirow{2}{*}{$\begin{array}{c}\text { Transtornos alimentares } \\
\text { Média }(D P)\end{array}$} & \multirow{2}{*}{$\begin{array}{l}\text { Obesidade } \\
\text { Média }(D P)\end{array}$} & \multirow{2}{*}{$\begin{array}{c}\text { População geral } \\
\text { Média }(D P)\end{array}$} & \multicolumn{2}{|c|}{ ANOVA } \\
\hline & & & & $F$ & $p$ \\
\hline Personalidade & - & - & - & - & - \\
\hline Neuroticismo & $1,58(0,66)$ & $1,68(0,66)$ & $1,30(0,61)$ & 1,77 & 0,176 \\
\hline Extroversão & $1,31(0,29)$ & $1,44(0,35)$ & $1,30(0,41)$ & 1,47 & 0,234 \\
\hline Estratégias de Coping & - & - & - & - & - \\
\hline Analise lógico & $12,49(3,56)$ & $12,83(3,29)$ & $12,41(3,01)$ & 0,16 & 0,848 \\
\hline Reavaliação positiva & $10,07(3,76)$ & $12,10(4,19)$ & $11,57(3,96)$ & 2,17 & 0,119 \\
\hline Busca de guia & $10,60(3,69)$ & $11,83(4,57)$ & $10,29(3,51)$ & 1,54 & 0,220 \\
\hline Resolução de prob. & $11,19(3,78)$ & $12,60(7,33)$ & $11,81(3,92)$ & 0,58 & 0,559 \\
\hline Evitação cognitiva & $10,73(3,99)$ & $10,07(7,05)$ & $10,66(4,04)$ & 0,17 & 0,846 \\
\hline Aceitação/resignação & $7,97(4,34)$ & $7,67(3,99)$ & $8,20(3,61)$ & 0,17 & 0,841 \\
\hline Busca de gratificação & $8,34(4,28)$ & $9,93(4,40)$ & $10,27(4,20)$ & 1,99 & 0,142 \\
\hline Descarga emocional & $10,03(4,07)$ & $9,00(3,89)$ & $8,52(3,07)$ & 1,65 & 0,197 \\
\hline EAT-26 & $31,77(12,23)$ & $22,80(8,96)$ & $11,87(6,90)$ & 42,56 & 0,001 \\
\hline Dieta & $19,73(10,00)$ & $14,03(6,12)$ & $6,61(5,24)$ & 31,94 & 0,000 \\
\hline Bulimia & $5,73(3,24)$ & $4,97(3,00)$ & $1,84(2,09)$ & 23,06 & 0,000 \\
\hline Controle oral & $6,30(3,77)$ & $3,80(3,35)$ & $3,50(2,19)$ & 10,21 & 0,000 \\
\hline
\end{tabular}

Apesar de verificar uma maior pontuação na escala de neuroticismo e extroversão na amostra de obesidade, as diferenças não foram significativas, como consta na Ta- bela 1. Também não houve diferenças significativas entre o uso de estratégias de coping segundo o tipo de população estudada. 
Tomaz, R. \& Zanini , D. S. (2009). Personalidade e Coping em Pacientes com Transtornos Alimentares e Obesidade.

Contudo, verifica-se uma diferença significativa nas médias do EAT-26 ( $F=42,56$ e $p=0,001)$, segundo o tipo de amostra: transtornos alimentares $(M=31,77 \mathrm{e}$ $D P=12,23)$, obesidade $(M=22,80$ e $D P=8,96)$ e população geral $(M=11,87$ e $D P=6,90)$.

Em uma análise mais detalhada por escalas, percebe-se que essa diferença ocorre nas escalas da dieta e de preocupação com os alimentos e bulimia, em que as médias foram maiores na população clínica, conforme demonstrado na Tabela 1. Porém, na escala do controle oral, a maior média observada foi em pacientes com transtornos alimentares, comparados com obesos e com a população geral.

\section{Relação entre Personalidade, Coping e Atitudes Alimentar}

Para avaliar a relação entre atitudes alimentares, estratégias de coping e perfil psicológico, utilizou-se a correlação bivariada de Pearson, que demonstrou correlações significativas, apresentadas na Tabela 2.
De acordo com a Tabela 2 verifica-se escassas relações entre coping, personalidade e atitudes alimentares. Contudo, parece que os individuos com alto índice de extroversão utilizam mais estratégias do tipo reavaliação positiva $(r=0,250)$, busca de guia $(r=0,210)$ e busca de gratificação alternativa $(r=0,287)$ enquanto indivíduos altos em neuroticismo buscam menos gratificação alternativa $(r=-0,213)$ como estratégia para enfrentar seus problemas. Por outro lado, quanto maior a pontuação geral na escala de atitudes alimentares maior o uso de estratégias do tipo descarga emocional $(r=0,219)$ e maior a pontuação em neuroticismo $(r=0,204)$.

A partir dos dados obtidos no estudo correlacional e para avaliar se a relação existente entre o neuroticismo, a descarga emocional explica a presença de atitudes alimentares de risco, foi conduzida uma regressão linear, cuja variável dependente foi o EAT-26 e os fatores neuroticismo e descarga emocional. Os dados estão demonstrados na Tabela 3.

Tabela 2

Correlação de Pearson entre Coping, Atitudes Alimentares e Perfil Psicológico

\begin{tabular}{lccc}
\hline \multicolumn{1}{c}{ Variáveis } & EAT-26 & Extroversão & Neuroticismo \\
\hline Análise lógica & 0,041 & 0,149 & $-0,038$ \\
Reavaliação positiva & $-0,115$ & $0,259^{* *}$ & $-0,151$ \\
Apoio social & 0,063 & $0,210^{*}$ & $-0,158$ \\
Resolução do problema & $-0,008$ & 0,124 & $-0,124$ \\
Evitação cognitiva & 0,113 & $-0,160$ & 0,005 \\
Aceitação resignação & 0,104 & $-0,023$ & 0,173 \\
Busca de gratificação & $-0,134$ & $0,287^{* *}$ & $-0,213^{*}$ \\
Descarga emocional & $0,219^{*}$ & 0,042 & 0,062 \\
Neuroticismo & $0,204^{*}$ & $-0,112$ & - \\
Extroversão & $-0,058$ & - & $-0,112$ \\
\hline
\end{tabular}

Nota. *Correlação significativa a 0,05 (2-tailed); **Correlação significativa a 0,01 (2-tailed).

Tabela 3

Regressão Linear entre Atitude Alimentar*, Coping (Descarga Emocional) e Personalidade (Neuroticismo)

\begin{tabular}{llll}
\hline & Beta & $\mathrm{T}$ & $\mathrm{p}$ \\
\hline Neuroticismo & 0,192 & 2,022 & 0,046 \\
Descarga Emocional & 0,207 & 2,183 & 0,031 \\
Coeficiente de Regressão & & \\
Variância Explicada & & \\
Teste Estatístico & $R=0,291$ & \\
& $R^{2}=0,085 ; R^{2}{ }_{\text {Ajustado }}=0,067$ & \\
& $F=4,708 ; p=0,011$ & \\
\hline
\end{tabular}

Nota. *EAT-26: variável dependente.

Conforme descrito na Tabela 3, os indivíduos que apresentam traço de personalidade neuroticismo e fazem uso da descarga emocional ou ventilação das emoções para enfrentar seus problemas, possuem mais atitudes alimen- tares inadequadas $(R=0,291, p=0,011)$, o que os caracteriza como portadores de distúrbios alimentares, segundo a classificação do EAT-26. 


\section{Discussão}

De acordo com os dados obtidos na comparação de média entre a escala de personalidade e a população (geral X clínica), não existe uma diferença significativa no perfil psicológico dos participantes da pesquisa, em relação aos fatores neuroticismo e extroversão. Apesar de diversos estudos apontarem uma associação entre uma maior pontuação em neuroticismo e diferentes populações clínicas (Costa, Sommerfield, \& McCrae, 1996), na pesquisa presente tal diferença não foi percebida.

Além disso, os dados demonstraram que os pacientes diagnosticados com transtornos alimentares e com obesidade não apresentam diferenças significativas entre o uso de estratégias de coping, quando comparados com a população geral. Estudos demonstram que pacientes com transtornos mentais, ou com alguma patologia referida, tendem a apresentar, significativamente, mais estratégias de coping evitativas que a população geral (Gregor, 2005; Tobin, 2004). No entanto, as diferenças entre as médias encontradas neste estudo foram mínimas e não significativas, estatisticamente. Pode-se postular que o tamanho reduzido da amostra clínica utilizada tenha contribuído para minimizar o efeito estatístico dessa diferença entre grupos. Contudo, vale ressaltar também que, apesar de não significativa, a diferença de médias entre as diferentes populações, observou-se que pacientes com transtornos alimentares e com obesidade utilizam mais a estratégia de coping descarga emocional (no sentido de chorar, gritar ou expressar comportamentalmente suas emoções sobre objetos ou pessoas) que a população geral. Esse dado confirma os achados de estudos anteriores (Moos, 1993), que demonstram uma forte associação entre descarregar emocionalmente os sentimentos quando do enfrentamento de um problema e sua maior repercussão na saúde mental.

Por outro lado, a avaliação da amostra em resposta ao instrumento EAT - 26 demonstra boa adequação do instrumento como medida avaliativa de transtorno alimentar e de obesidade. O presente estudo demonstrou que sujeitos da população geral apresentaram pontuações significativamente mais baixas no EAT - 26 que pacientes com diagnóstico de transtorno alimentar e de obesidade. Isso confirma a aplicabilidade deste teste e seu valor discriminativo em termos de diagnóstico de tais transtornos, conforme já mencionado em estudos anteriores (Bighetti, 2003; Nunes, 2005). Isso justifica o fato de este ter se tornado o teste mais utilizado para avaliação de transtornos alimentares nos ultimos tempos (Bighetti, 2003), além de sua fácil aplicabilidade e diagnóstico, o que favorece a descoberta das patologias, possibilitando uma melhor intervenção em períodos ainda precoces do transtorno.

Por último cabe, ressaltar que os transtornos alimentares parecem ser influenciados por fatores de personalidade, tais como o neuroticismo, e por formas de enfrentamento a problemas, tais como descarga emocional. Isso sugere que indivíduos inseguros, com labilidade emocional, irritadiços, nervosos, com dificuldade para lidar com estresses e que, ao enfrentarem problemas, tendem a descarregar emocionalmente seus sentimentos, com o objetivo de diminuir a ansiedade causada pelo problema, mas não direcionando ações para a sua resolução, tendem a desenvolver mais transtornos alimentares e obesidade que a população geral. Esse dado sugere a necessidade de avaliação deste perfil psicológico e de enfrentamento a problema como um fator de risco para o desenvolvimento de problemas psicológicos no geral, conforme demonstra Zanini (2003), e para o desenvolvimento de transtornos alimentares, mais especificamente conforme demonstrado neste estudo.

De acordo com o objetivo geral deste trabalho, observou-se que pacientes com transtornos alimentares e com obesidade apresentam um perfil psicológico e o uso de estratégias de coping desadaptadas em medidas diferentes daquelas utilizadas pela população geral.

Assim, pode-se postular a necessidade de que as intervenções psicológicas orientadas para o atendimento dessa população direcionem suas metas para o desenvolvimento de habilidades de coping que permitam ampliar o repertório comportamental referente às formas de enfrentamento a problemas e possam gerar mudanças nas formas de percepção e relação com o meio, assim como maior assertividade e demais características pessoais que busquem fomentar maior auto-controle emocional e autoconfiança como estratégias de ampliação de repertório comportamental para combater às características do traço de personalidade neuroticismo.

Contudo, estudos posteriores devem ser conduzidos com um número maior de sujeitos com diagnósticos e com distinção dos tipos de transtornos alimentares, a fim de avaliar uma possível variação no uso de coping e perfil de personalidade, em função do tipo de transtorno alimentar diagnosticado.

\section{Referências}

American Psychiatric Association. (1995). Manual diagnóstico e estatístico de transtornos mentais IV-TR. Porto Alegre, RS: Artes Médicas.

American Psychiatric Association. (2002). Practice guideline for the treatment of patients with eating disorders (Revision). American Journal of Psychiatry, 157, 1-39.

Bighetti, F. (2003). Tradução e validação do Eating Attitudes Test (EAT-26) em adolescentes do sexo feminino na cidade de Ribeirão Preto-SP. Dissertação de Mestrado nãopublicada, Universidade de São Paulo, Ribeirão Preto, SP.

Bighetti, F., Santos, J. E., \& Ribeiro R. P. P. (2006). Grupo de orientação clínico-nutricional a familiares de portadores de transtornos alimentares: Uma experiência "GRATA". Medicina Ribeirão Preto, 39, 410-414.

Binford, R. B., Crosby, R. D., Mitchell, J. E., Mussell, M. P., Peterson, C. B., \& Crow, S. J. (2005). Coping strategies in bulimia nervosa treatment: Impact on outcome in group cognitive-behavioral therapy. Journal of Consulting and Clinical Psychology, 73, 1089-1096. 
Blumenthal, J. A., Babyak, M. A., Carney, R. M., Keefe, F. J., Davis, R. D., \& LaCaille, R. (2006). Telephone-based coping skills training for patients awaiting lung transplantation: The INSPIRE study. Journal of Consulting and Clinical Psychology, 74, 535-544.

Connor-Smith, J. K., \& Flachsbart, C. (2007). Relations between personality and coping: A meta-analysis. Journal of Personality and Social Psychology, 93, 1080-1107.

Cordás, T. A., \& Neves, J. E. P. (2000). Escalas de avaliação de transtornos alimentares. In C. Gorenstein, L. H. S. G. Andrade, \& A. W. Zuardi (Eds.), Escalas de avaliação em psiquiatria e psicofarmacologia (pp. 345-347). São Paulo, SP: Lemos.

Cordás, T. A., \& Weinberg, C. (2002). Santas anoréxicas na História do Ocidente: O caso de Santa Maria Madalena de Pazzi. Revista Brasileira de Psiquiatria, 24, 157-158.

Costa, P. T., Somerfield, M. R., \& McCrae, R. R. (1996). Personality and health. In L. Pervin (Ed.), Handbook of personality theory and research (pp. 44-61). London: Guilford Press.

Dessen, M. C. (2005). Bem-estar pessoal nas organizações: $O$ impacto de configurações de poder e características de personalidade. Dissertação de Mestrado não-publicada, Universidade de Brasília, DF.

Dobrow, I. J., Kamenetz, C., \& Devlin, M. J. (2002). Aspectos psiquiátricos da obesidade. Revista Brasileira de Psiquiatria, 24, 63-67.

Freitas, S., Gorenstein, C., \& Appolinario, J. C. (2002). Instrumentos para a avaliação dos transtornos alimentares. Revista de Psiquiatria, 24, 34-38.

Furtado, E. S., Falcone, E. M. O., \& Clark, C. (2003). Avaliação do estresse e das habilidades sociais na experiência acadêmica de estudantes de medicina de uma universidade do Rio de Janeiro. Interação em Psicologia, 7, 43-51.

Fyer, S., Waller, G., \& Stenfert-Kroese, B. (1997). Stress, coping and disturbed eating attitudes in teenage girls. International Journal of Eating Disorders, 22, 427-436.

Galaif, E. R., Sussman, S., Chou, C., \& Wills, T. A. (2003). Longitudinal relations among depression, stress, and coping in risk youth. Journal of Youth and Adolescence, 32, 243258.

Gómez-Fraguela, J. A., Luengo-Martín, A., Romero-Triñanes, E., Villar-Torres P., \& Sobral-Fernández, J. (2006). Estratégias de afrontamiento en el inicio de la adolescencia y su relación con el consumo de drogas y la conducta problemática. International Journal of Clinical and Health Psychology, 6, 581-597.

Gongora, V. C., van de Staak, C. P. F., \& Derksen, J. J. L. (2004). Personality disorders, depression and coping styles in the Argentinean bulimic patients. Journal of Personality Disorders, 18, 272-285.

Gorgati, S. B., Holcberg, A. S. \& Oliveira, M. D. (2002). Abordagem psicodinâmica no tratamento dos transtornos alimentares. Revista Brasileira de Psiquiatria, 24, 44-48.

Gregor, M. M. (2005). The effect of acceptance and nonacceptance based coping strategies on symptom severity in the Eating Attitudes Test (EAT). Unpublished doctoral dissertation, Faculty of the Chicago, School of Professional Psychology, Chicago, IL.

Griffith, M. A., Dubow, E. F., \& Ippolito, M. F. (2000). Developmental and cross-situational differences in adolescent's coping strategies. Journal of Youth and Adolescence, 29, 183-197.
Guedea, M. T. D., Albuquerque, F. J. B., Tróccoli, B. T., Noriega, J. A. V., Seabra, M. A. B., \& Guedea, R. L. D. (2006). Relação do bem-estar subjetivo, estratégias de enfrentamento e apoio social em idosos. Psicologia: Reflexão e Crítica, 19, 301-308.

Hay, P. J. (2002). Epidemiologia dos transtornos alimentares: Estado atual e desenvolvimentos futuros. Revista de Psiquiatria, 24, 13-17.

Jonge, J., \& Dormann, C. (2006). Stressors, resources, and strain at work: A Longitudinal Test of the Triple-Match Principle. Journal of Applied Psychology, 91, 1359-1374.

King, E. B., Shapiro, J. R., Hebl, M. R., Singletary, S. L., \& Turner, S. (2006). The stigma of obesity in customer service: A mechanism for remediation an bottom-line consequences of interpersonal discrimination. Journal of Applied Psychology, 91, 579-593.

Lazarus, R. S., \& Folkman, S. (1984). Stress, appraisal and coping. New York: Springer.

Margis, R., Picon, P., Cosner, A. F., \& Silveira, R. O. (2003). Relação entre estressores, estresse e ansiedade. Revista de Psiquiatria do Rio Grande do Sul, 25, 65-74.

Magalhães, V. C., \& Mendonça, S. (2005). Transtornos alimentares em universitárias: Estudo de confiabilidade da versão brasileira de questionários autopreenchíveis. Revista Brasileira de Epidemiologia, 8, 236-245.

Moos, R. H. (1993). Coping Response Inventory Adult Form: Professional manual. Odessa, FL: PAR Psychological Assessment Resources.

Moos, R. H. (2002). The mystery of human context and coping: An unravelling of clues. American Journal of Community Psychology, 30(1), 67-88.

Moos, R. H. (2003). Social context: Transcending their power and their fragility. American Journal of Community Psychology, 31(2), 1-13.

Nunes, M. A. A., Appolinario, J. C., Abuchaim, A. L. G., \& Coutinho, W. (2006). Transtornos alimentares e obesidade. Porto Alegre, RS: Artmed.

Nunes, C. H. S. S. (2005). Construção, normatização e validação das escalas de socialização e extroversão no modelo dos Cinco Grandes Fatores. Tese de Doutorado nãopublicada, Instituto de Psicologia, Universidade Federal do Rio Grande do Sul, Porto Alegre, RS.

Organização Mundial de Saúde. (1993). Classificação estatística internacional de doenças e problemas relacionados à saúde. Porto Alegre, RS: Artes Médicas.

Pasquali, L., Azevedo, A. M., \& Ghesti, I. (1997). Manual Técnico do Inventário Fatorial de Personalidade. São Paulo, SP: Casa do Psicólogo.

Pinheiro, A. P., Sullivan, P. F., Bacaltchuck, J., Prado-Lima, P. A. S., \& Bulik, C. M. (2006). Genética em transtornos alimentares: Ampliando os horizontes de pesquisa. Revista Brasileira de Psiquiatria, 28, 218-225.

Pitanga, F. J. G., \& Lessa, I. (2007). Associação entre indicadores antropométricos de obesidade e risco coronário em adultos na cidade de Salvador. Revista Brasileira de Epidemiologia, 10, 239-248.

Rebelo, A., \& Leal, I. (2007). Fatores de personalidade e comportamento alimentar em mulheres portuguesas com obesidade mórbida: Estudo exploratório. Análise Psicológica, 3, 467-477.

Rowe, M. M. (2006). Four-year longitudinal study of behavioral changes in coping with stress. American Journal Health Behavior, 30, 602-612. 
Rydén, A., Karlsson, J., Persson, L., \& Sjöström, L. (2001). Obesity-related coping and distress and relationship to treatment preference. Journal of Clinical Psychology, 40, 177-188.

Schat, A. C. H., Kelloway, E. K., \& Desmarais, S. (2005). The Physical Questionnaire (PHQ): Construct validation of a SelfReport Scale of Somatic Symptoms. Journal of Occupational Health Psychology, 10, 363-381.

Shapiro, J. R., King, E. B., \& Quiñones, M. A. (2007). Expectations of obese trainees: How stigmatized trainee characteristics influence training effectiveness. Journal of Applied Psychology, 92, 239-249.

Sorkin, D. H., \& Rook, K. S. (2006). Dealing with negative social exchanges in later life: Coping response, goals, and effectiveness. Psychology and Aging, 21, 715-725.

Teichmann, L., Olinto, M. T. A., Costa, J. S. D., \& Ziegler, D. (2006). Fatores de risco associados ao sobrepeso e à obesidade em mulheres de São Leopoldo, RS. Revista Brasileira de Epidemiologia, 9, 360-373.

Tobin, D. L. (2004). Terapia de estratégias para combater a bulimia nervosa. São Paulo, SP: Roca.

Tróccoli, B. T., Vasconcelos, T. S., Araújo R. M., \& Meiçó, C. (2001). Inventário Reduzido dos Cinco Grandes Fatores de Personalidade [Resumo]. In Universidade Federal da Bahia (Ed.), Anais do II Congresso Norte Nordeste de Psicologia. Salvador, BA: Editora da Universidade Federal da Bahia.

Velásquez-Meléndez, G., Gazzinelli, A., Côrrea-Oliveira, R., Pimenta, A. M., \& Kac, G. (2007). Prevalence of metabolic syndrome in a rural área of Brazil. São Paulo Medical Journal, 125, 155-162.

Wilson, G. T., \& Fairburn, C. (1993). Cognitive treatments for eating disorders. Journal of Consulting and Clinical Psychology, 61, 261-269.

Zanini, D. S. (2003). Coping: influencia de la personalidad y repercusiones en la salud mental de los adolescentes. Unpublished doctoral dissertation, Universidad de Barcelona, España. 